Dans le présent article, I'OFSP rappelle une nouvelle fois de manière claire et précise les conditions requises en matière de formation prégraduée et postgraduée afin de pouvoir pratiquer la radiologie conventionnelle dans le domaine des doses modérées. Ces informations concernent les médecins et les assistantes médicales CFC. Toutes et tous les collègues qui exécutent des radiographies conventionnelles dans le domaine des doses modérées ou les font exécuter par leur assistante médicale CFC sont cordialement invités à lire avec attention ces informations de I'OFSP.

\title{
La radiologie conventionnelle dans le domaine des doses modérées
}

\author{
Nicolas Stritt ${ }^{\mathrm{a}}$, Thomas Flury ${ }^{\mathrm{b}}$, Annemarie Harwig ${ }^{\mathrm{c}}$ \\ ${ }^{a}$ Dr phil. nat., chef de section, Installations de recherche et médecine nucléaire; ${ }^{b}$ Dr phil. nat., collaborateur scientifique; ${ }^{c}$ Collaboratrice scientifique
}

En vertu de l'ordonnance sur la radioprotection, l'utilisation d'appareils de radiologie à des fins médicales est réservée aux catégories professionnelles ayant une formation spécifique en radioprotection. La formation requise pour acquérir ces compétences est différente selon que les clichés réalisés relèvent du domaine des doses faibles, des doses modérées ou des doses élevées. Cet article présente l'enquête que l'OFSP s'apprête à mener et donne une vue d'ensemble des formations et des compétences dont doivent justifier les médecins et le personnel médical pratiquant la radiologie diagnostique conventionnelle. Vous trouverez des informations détaillées sur l'enquête de l'OFSP ainsi que sur les formations et les formations complémentaires à l'adresse suivante: www.bag.admin.ch/rx-cabinets-medicaux

\section{Audit dans les cabinets médicaux}

Il y a quelques années, la division Radioprotection de l'Office fédéral de la santé publique (OFSP) a soumis une centaine d'établissements à un audit [1]. Cette campagne a donné l'occasion de sensibiliser les utilisateurs à des aspects importants de la radioprotection et à la formation requise en la matière. L'analyse des données recueillies a montré que les médecins comme les assistants médicaux (AM) devraient suivre la formation complémentaire en radioprotection requise par la législation afin de réaliser des clichés dans le domaine des doses modérées (examens radiologiques conventionnels de l'abdomen, de la colonne vertébrale et du bassin). Il a été constaté en particulier que plus de $80 \%$ des AM qui prennent des clichés en utilisant des techniques radiologiques conventionnelles élargies n'ont pas suivi la formation nécessaire.

Une qualification et une formation adéquate permettent de garantir une radioprotection plus efficace

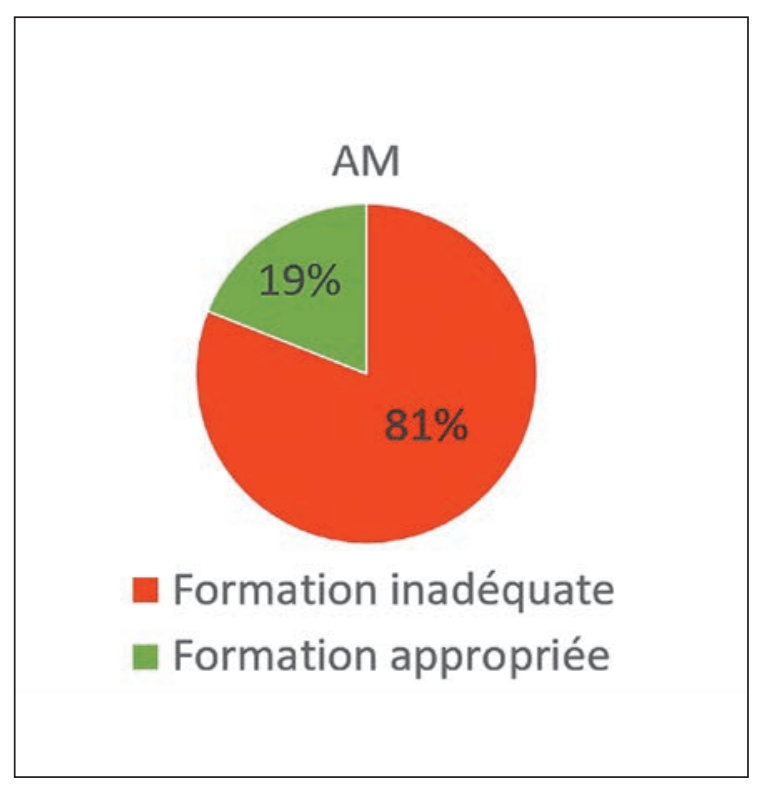

Figure 1: Formation aux techniques radiologiques conventionnelles élargies dans les cabinets médicaux audités. 
et d'optimiser les doses délivrées aux patients lors des examens diagnostiques. Il est donc important pour la protection des patients que tout médecin exerçant en cabinet qui demande et analyse des clichés réalisés dans le domaine des doses modérées soit titulaire de l'attestation de formation complémentaire «Examens radiologiques à fortes doses (CMPR)» [2] correspondant à son activité. De plus, le cabinet doit compter au moins un AM ayant suivi une formation complémentaire reconnue dans le domaine des techniques radiologiques conventionnelles élargies pour effectuer les clichés.

\section{Formation complémentaire pour les techniques radiologiques conventionnelles élargies}

La formation de base des AM ( 3 ans) les habilite à utiliser des installations radiologiques dans le domaine des doses faibles (thorax et extrémités), sur prescription d'un médecin ayant la qualité d'expert.

L'exigence d'une formation complémentaire en radioprotection, requise pour la prise de clichés dans le domaine des doses modérées, a été reprise telle quelle dans la révision de l'ordonnance sur la radioprotection [3] et l'ordonnance sur la formation en radioprotection [4] du 26 avril 2017 entrée en vigueur le $1^{\text {er }}$ janvier 2018. Les 40 unités d'enseignement portent sur les techniques de positionnement pour la prise de clichés dans les régions de l'abdomen, de la colonne vertébrale et du crâne, et sur l'interprétation des paramètres de qualité des clichés (contraste, définition). Cette formation complémentaire donne la qualification nécessaire en

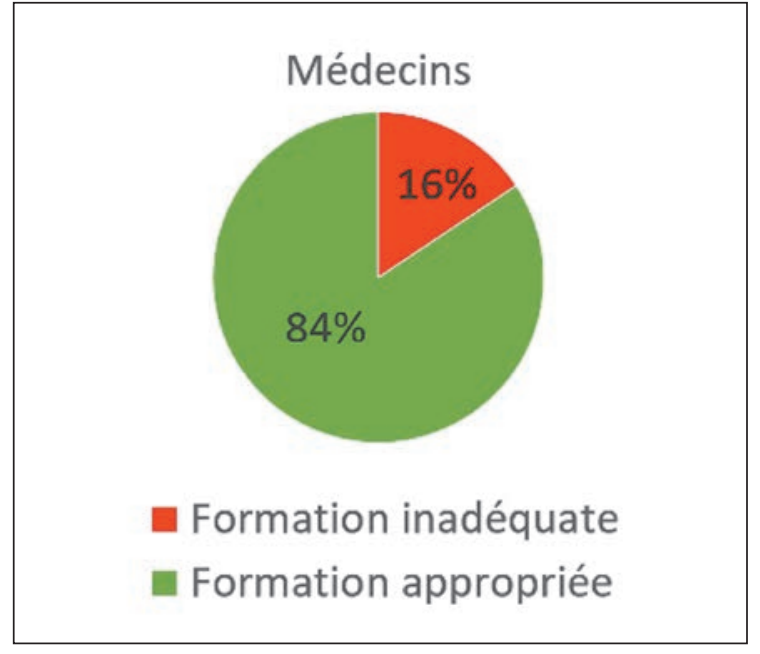

Figure 2: Qualification des médecins dans les cabinets médicaux audités.

radioprotection et habilite les AM à utiliser des installations radiologiques dans les domaines des doses modérées et des doses faibles sur prescription d'un médecin expert. Elle ne permet par contre pas à un AM d'effectuer des examens CT, des radioscopies ou des mammographies.

\section{Attestation de formation complémentaire $(\mathrm{AFC})$ «Examens radiologiques à fortes doses (CMPR)»}

L'audit a également montré qu'environ 20\% des médecins ne possédaient pas la formation complémentaire requise par la législation en radioprotection en vigueur.

Tableau 1: Activités autorisées et non autorisées en radiologie selon la formation de base et la formation complémentaire du personnel médical.

\begin{tabular}{|c|c|c|}
\hline & Radiologie conventionnelle & \\
\hline $\begin{array}{l}\text { Catégorie professionnelle } \\
\text { (abréviations selon I'ordonnance sur la formation) }\end{array}$ & $\begin{array}{l}\text { Domaine des doses faibles: } \\
\text { thorax et extrémités }\end{array}$ & $\begin{array}{l}\text { Domaine des doses modérées: } \\
\text { abdomen, colonne vertébrale, } \\
\text { crâne }\end{array}$ \\
\hline $\begin{array}{l}\text { MP } 7 \\
\text { AM avec CFC } \\
\text { MP } 9 \\
\text { Autres catégories de personnel médical avec for- } \\
\text { mation reconnue par l'OFSP habilitant à prendre } \\
\text { des clichés du thorax et des extrémités }\end{array}$ & $\begin{array}{l}\qquad \sqrt{ } \\
\text { Manipulation de l'installation } \\
\text { sur prescription d'un médecin } \\
\text { expert }\end{array}$ & $\begin{array}{c}\text { X } \\
\text { Non autorisé sans formation } \\
\text { complémentaire dans le domaine } \\
\text { des techniques radiologiques } \\
\text { conventionnelles élargies }\end{array}$ \\
\hline $\begin{array}{l}\text { MP } 8 \\
\text { AM avec CFC et avec une formation complémen- } \\
\text { taire dans le domaine des techniques radiologiques } \\
\text { conventionnelles élargies } \\
\text { Autres catégories de personnel médical habilitées } \\
\text { à prendre des clichés du thorax et des extrémités } \\
\text { avec une formation complémentaire dans le } \\
\text { domaine des techniques radiologiques conven- } \\
\text { tionnelles élargies }\end{array}$ & $\begin{array}{l}\qquad \sqrt{ } \\
\text { Manipulation de l'installation } \\
\text { sur prescription d'un médecin } \\
\text { expert }\end{array}$ & $\begin{array}{l}\qquad \sqrt{ } \\
\text { Manipulation de l'installation } \\
\text { sur prescription d'un médecin } \\
\text { expert }\end{array}$ \\
\hline $\begin{array}{l}\text { MP 4/6 } \\
\text { Techniciens en radiologie médicale ES/HES (TRM) }\end{array}$ & $\begin{array}{l}\qquad \sqrt{ } \\
\text { Manipulation de l'installation sur } \\
\text { prescription d'un médecin expert }\end{array}$ & $\begin{array}{l}\qquad \sqrt{ } \\
\text { Manipulation de l'installation sur } \\
\text { prescription d'un médecin expert }\end{array}$ \\
\hline
\end{tabular}


Tableau 2: Formation complémentaire requise par les législations pour les médecins afin d'acquérir des compétences dans le domaine des doses modérées (bassin, hanches, abdomen, colonne vertébrale).

\begin{tabular}{|c|c|c|}
\hline & Radiologie conventionnelle & \\
\hline $\begin{array}{l}\text { Titres de spécialiste } \\
\text { (abréviations selon l'ordonnance } \\
\text { sur la formation) }\end{array}$ & $\begin{array}{l}\text { Domaine des doses faibles: } \\
\text { thorax et extrémités }\end{array}$ & $\begin{array}{l}\text { Domaine des doses modérées: } \\
\text { abdomen, colonne vertébrale, crâne }\end{array}$ \\
\hline $\begin{array}{l}\text { MA } 11 \\
\text { Tous les médecins }\end{array}$ & $\begin{array}{l}\sqrt{ } \\
\text { Justification, réalisation et évaluation } \\
\text { de clichés radiologiques conventionnels }\end{array}$ & $\begin{array}{c}\mathbf{x} \\
\text { Non autorisé sans AFC «Examens } \\
\text { radiologiques à fortes doses» }\end{array}$ \\
\hline
\end{tabular}

L'acquisition des compétences en radiographie dans le domaine des doses modérées requiert les formations complémentaires indiquées dans le tableau ci-dessus pour chaque titre postgrade fédéral pour les médecins. Le titulaire de l'autorisation est garant des personnes (médecins et personnel médical) qu'il emploie pour réaliser les clichés: il s'assure qu'elles sont qualifiées et vérifie qu'elles possèdent les qualifications complémentaires requises dans le domaine des techniques radiologiques conventionnelles élargies.

\section{Enquête en ligne pour évaluer la situation actuelle en matière de formation dans les cabinets médicaux}

Fort de ce constat, l'OFSP avait écrit, il y a quelques années, aux 3500 cabinets médicaux équipés d'une installation radiologique en leur expliquant ces lacunes dans la formation et que celle-ci devait être rattrapée. La division Radioprotection de l'OFSP souhaite évaluer dans quelle mesure la situation s'est améliorée et sur quels aspects il convient de poursuivre les efforts. $\mathrm{Au}$ cours des mois à venir, elle fera parvenir progressivement aux cabinets médicaux équipés d'une installation de radiologie une invitation écrite à participer à une enquête en ligne afin d'obtenir des informations sur les aspects suivants:
- situation en matière de formation des médecins qui effectuent ou évaluent des clichés radiologiques;

- situation en matière de formation du personnel qui manipule les installations de radiologie dans le domaine des doses faibles (thorax et extrémités) et dans le domaine des doses modérées (abdomen, colonne vertébrale et bassin);

- statistique du nombre de clichés effectués par mois.

\section{Informations}

L'OFSP publie des informations sur la formation et les formations complémentaires requises ainsi que sur les activités autorisées en matière de radioprotection et donne des précisions sur l'enquête en cours à l'adresse suivante: www.bag.admin.ch/rx-cabinets-medicaux

\section{Références}

1 Rapport final: Radiologie numérique dans les cabinets médicaux: https://www.bag.admin.ch/bag/fr/home/gesetze-undbewilligungen/gesuche-bewilligungen/bewilligungen-aufsichtim-strahlenschutz/schlussberichte-aufsichtsschwerpunkte.html

2 Informations complémentaires concernant l'attestation de formation complémentaire (AFC): www.kollegium.ch

3 Informations complémentaires concernant l'ordonnance sur la radioprotection (ORaP): https://www.admin.ch/opc/fr/ classified-compilation/20163016/index.html

4 Informations complémentaires concernant l'ordonnance sur les formations et les activités autorisées en matière de radioprotection: https://www.admin.ch/opc/fr/classified-compilation/ 20163019/index.html 